\title{
IMMUNOLOGICAL STUDIES ON THE MECHANISM OF RESISTANCE IN EXPERIMENTAL ONCHOCERCIASIS
}

\author{
D.O. Akinboye \\ Department of Zoology \\ University of Ibadan, Ibadan, Nigeria \\ E-mail: akinboye@skannet.com
}

\begin{abstract}
Human onchocerciasis is a debilitating disease, which causes lymphatic obstruction and gives rise to genital elephantiasis. The microfilariae also cause severe itching, while invasion of the eyes by large numbers, and the reaction to their presence, lead to river blindness. This results in severe medical, social and economic problems (Stevenson 1987).The implications of the disease bring about the need to control onchocerciasis by understanding the mechanism of the host's resistance.
\end{abstract}

The aim of this study was to investigate the possibility of using laboratory rodents as models for immunological studies of onchocerciasis. Living adult Onchocerca (O.) armillata and $\underline{\text { O. volvulus }}$ were surgically transplanted into the peritoneal cavities of rats, after initial immunizations or transplantations, in order to investigate the survival rates of the transplanted parasites. Steroid

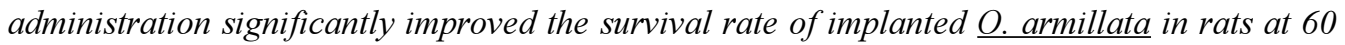
days $\left(X^{2}=3, d f=1, P<0.05\right)$. A similar effect was produced in $\underline{O}$. volvulus with the survival rate being significantly higher than in rats not treated with cortisone $\left(x^{2}=6.3, d f=1, P<0.01\right)$.

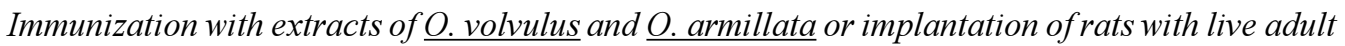
worms hindered the survival of homologous implants significantly, while no such effect was produced in heterologous implants. Immunization with microfilariae of either species of worms had no effect on the survival rate of subsequent implants of adult worms of the same species. Immunization with the peritoneal exudates, obtained from rats previously implanted with adult

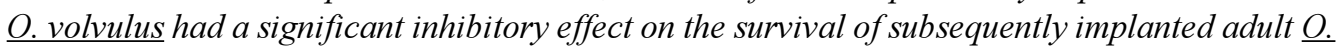
volvulus $\left(X^{2}=27, d f=1, P<.001\right)$. The model is valuable for immunological and chemotherapeutic studies of the two species of Onchocerca.

\section{INTRODUCTION}

Onchocerciasis is a helminthic disease of man and animals, which occurs mainly in the tropical regions of the world. According to the World Health Organization (W.H.O. 1973), human onchocerciasis is restricted to Africa and parts of Central and Southern America. The endemic foci of human Onchocerciasis, caused, by Onchocerca volvulus, exist in tropical Africa, Central and Southern America, Yemen Arab Republic and Mexico. In Yemen, Onchocerciasis is endemic in the southern regions around Taiz. In Latin America, onchocerciasis is endemic in Mexico, Guatemala, Colombia, Venezuela and Brazil, W.H.O. (1976). The same authors also estimated that about 50 million people were affected by onchocerciasis and about
1 million were blind, as a result of the disease. In man, the disease is characterized by dermatitis, lymphadenopathy, pruritis and ocular lesions, which often lead to blindness.

The disease is caused by Onchocerca volvulus in man, and it is transmitted by black flies of the genus Simulium. Several Onchocerca species also occur in gorillas, cattle, waterbufaloes, donkeys, camels and African antelopes, (Noble and Noble, 1976). There have been occasional records of animal species in man, in North America, Europe and the U.S.S.R. These zoonotic infections are mainly due to Onchocerca cervicalis and Onchocerca gutturosa (Muller, 1978). 


\section{BACKGROUND TO THE STUDY}

Onchocerciasis is increasingly recognized as a major public health problem and a serious obstacle to socio-economic development, (Buck 1974, W.H.O. 1978). This emphasizes the need to control the disease in man, possibly by understanding the host's immune reaction to the parasite.

The mechanisms involved in immune response, in Onchocerciasis are poorly understood. However, natural resistance has been found to occur in all forms of animal onchocerciasis, except in man, gorilla and chimpanzee (WHO, 1987). In man, there seems to be no evidence of acquired immunity to re-infection. However, a high level of resistance to challenge infection has been demonstrated in mice with the microfilariae of $O$. lienalis, (Ivalions et al, 1984).

The effect of sensitization has been demonstrated in mice, with a range of heterologous species of Onchocerca, by Towson et al (1985). Using O. cervicalis microfilariae, the latter authors found that there was resistance to challenge, with $O$. lienalis microfilariae.

In this study, the influence of primary immunisation or implantation on the establishment of subsequent implants of adult $O$. armillata and $O$. volvulus were investigated. Findings from this study will enable immunologist to obtain more information on the establishment of Onchocerciasis in the human host, thereby contributing to control strategies of the disease.

\section{Life Cycle of Onchocerca Volvulus}

The microfilariae of Onchocerca volvulus measure about 150 to $350 \mu \mathrm{m}$ in length and are present in onchocercomata, in connective tissues, superficial lymphatic vessels and sometimes in urine of infected patients (Noble and Noble 1976).

The insect vector of Onchocerca is the black fly, Simulium. The larvae develop in fast flowing rivers and streams. The adult fly bites man and animals. When biting to feed, the vector fist cuts the skin by the rasping action of the bladelike mandibles(Cupp1996) through the epidermis, into the dermis and sucks up blood from a pool, formed on the skin. This pool of blood comprises of blood cells, tissues fluids and microfilariae (Connor and Neafie 1976).

Within the fly, the microfilariae migrate to the thoracic muscles and develop into an infective larva. The infective larvae then migrate into the proboscis of the vector. When the infected fly bites man, the infective larvae get into the human skin or subcutaneous connective tissues. The larvae then develop to mature worms, which appear in nodules within a year, and these nodules are usually found under the skin. The nodules measure about $1 \mathrm{~mm}$ to $80 \mathrm{~mm}$ in diameter. A light infection consists of a few nodules but sometimes in a heavy infection, as many as hundred nodules could be found. These nodules tend to lie over bony prominences like skull, scapulae, ribs, elbows, trochanters, iliac crest, sacrum and knees, (Connor and Neafie op cit).

\section{Pathogenesis of Onchocerciasis}

Most of the lesions of Onchocerciasis involve dermal and ocular tissues. Onchocerciasis does not appear to result from mechanical damage caused by the presence of adult worms or microfilariae per se but due to inflammatory reactions around microfilariae and dead or dying worms. Histopathological studies indicate that living microfilariae generally evoke no inflammatory reaction in the eye or in the dermis but cells, particularly eosinophils and macrophages, usually surround damaged microfilariae (Gibson et al., 1976; Henson et al., 1980).

According to Connor and Neafie (1976) patients who suffer from severe infections have millions of microfilariae migrating into the skin, the eye, lymph nodes, inner organs and blood streams, where they cause progressive and severe inflammatory lesions.

Onchocerca nodules usually contain several worms, surrounded by inflammatory reactions. This varies from suppurative to granulomatous, which progress to fibrosis, calcification and sometimes ossification. The nodules are usually painless, but the very large ones, which lie over pressure points and those undergoing degenerative changes, may become tender or even painful, (W.H.O., op, cit.) while any part of the skin, especially the feet, may have altered pigmentation. Persistent scratching may cause bleeding and lead to secondary infection. There could also be advanced changes in form of edema, papules, wrinkling, dermal thickening, scaling and greatly enlarged lymph nodes. According to Conner and Neafie (1976) African patients have slightly enlarged hard regional lymph and in long standing cases, those may progress to a hanging groin. The writer stated further, that a layer of fibroid materials sometimes covers the worm and may partially protect it from the host's immune reactions. They also noted that early changes in the dermis are minimal and 
characterised by a few proliferating fibroblasts, edema, and a few lymphocytes, histocytes, plasma cells and eosinophils around vessels and appendages. Mast cells may be increased but neutrophils are absent. Ogundipe et al., (1984) observed 3 types of reactions around $O$. armillata in cattle. The first type had walls that were massively infiltrated with Langahans type giant cells, epithelioid cells, eosinophils and proliferating fibroblasts. The second type was made up of little or no cellular infiltration of its wall, and adult worms were often surrounded by only proliferated fibrous tissue. The thirds type consisted of old tunnels, which contained dead or degenerated worms, which were often calcified and in some cases, were surrounded by eosinophils, plasma cells and fibrous tissues.

In West Africa, Onchocerciasis is a common cause of blindness, which is especially more prevalent in the Savannah than in the forest regions. The main damage is caused by the presence of living or dead microfilariae in the eye, (W.H.O. op. cit). The inflammatory foci, which surround microfilariae, lead to both punctate keratitis and corneal opacity (Ottensen 1984). The early sign of visual impairment is due to punctate keratitis and sclerotising keratitis. According to Anderson and Fonts op.cit, punctate keratitis sometimes causes photophobia and lacrymation. Such patients usually have no symptoms and visual impairment is minimal. The writers further stated that in villages where infections were severe, punctate keratitis was common in children under 10 years of age. Corneal opacity was due to the inflammatory reaction around degenerating, microfilariae, the body of which remained visible in the center of the lesion, for several days. These punctate lesions develop in all parts of the corneal but are most frequent at its periphery. Sclerotising keratitis is a stromal inflammation and a common cause of blindness in Central America and in the African Savanna. It is caused by the invasion of the cornea by numerous microfilariae. The lesion begins peripherally in the posterior chamber of the eye. The scarcity of human eyes for pathological examinations has severely limited studies on the histopathology of ocular onchocerciasis. Most of the human cases materials available were obtained from advanced stages in disease of many years' duration,( W.H.O.1982) . Exploration from these to the early stages of the disease process was difficult. Laboratory animals such as guinea pigs and rabbits have been used as models for investigating the pathogenic mechanism of eye lesions in onchocerciasis.

In the posterior chambers of the eye, lesion do not often correlate with microfilariae density (W.H.O. op.cit) suggesting that the pathogenic mechanism may be different from anterior chamber lesion. Visual loss appears to involve optic nerve damage and choroidoretinitis.

\section{Immunology of Onchocerciasis}

The mechanism involved in modulation of the immune response, in persons with chronic O.volvulus infection, are poorly understood (Gallin, et al 1988). However, natural resistance to $O$. volvulus appeared to occur in all animal species, except in human, gorilla and the chimpanzee (WHO1987). There seemed to be no evidence of acquired immunity to reinfection in humans. Patients whose infections have been cured but who continue to be exposed to heavy transmission in an endemic area rapidly become reinfected, and these infections may reach pre-treatment levels of intensity within 4 to nine years. In Onchocerciasis, there is elevation of total and specific $1 \mathrm{gG}$ and $1 \mathrm{gE}$ antibodies. The protective effect of the immunity however, is lacking even in the presence of demonstrable immune responses (Ambroise-Thomas 1980, Weiss et al., 1981). This is because filarial infections are typically chronic events as $O$. volvulus lives for up to 16 years in its host.

The response of the host to filarial parasites is critical for determining the clinical consequence of the disease and for immunodiagnosis. The specific antibody, cellular responses generated by the host and the seeming lack of responsiveness to the parasites are also important immunological phenomena.

Such unresponsiveness occurs, not because the patient fails to become sensitized to filarial antigens but because various modulating mechanisms develop, that can specifically suppress responsiveness to these antigens (Ottensen 1984). Similar studies of lymphocytes reactivity in other animal model systems have substantiated the finding of antigen-specific cell mediated immune suppression during chronic filariasis and have also indicated that suppressive mechanism develop, following a transient, initial phase of vigorous responsiveness to parasite antigens, that occur early in infection (Weiss, 1978; Portono et al., 1976 and Sisley, 1987) noted that there was parasite specific $1 \mathrm{gG}$ antibody concentration which was usually present in the sera of patients affected with Onchocerciasis

In an attempt to determine parasite specific immune response to Onchocerca lienalis microfilariae in normal and immunodeficient mice, Carlow, Dobinson and Bianco 
(1987) measured the levels of $1 \mathrm{gG} 1, \operatorname{lgG} 2 \mathrm{a}, 1 \mathrm{gG} 2 \mathrm{~b}, \operatorname{lgG} 3$, $1 \mathrm{gM}$ and $1 \mathrm{gA}$, which were reactive with microfilariae antigen. Antibody titers were greatest on the 25 th day and there was decreased level of $1 \mathrm{gM}, 1 \mathrm{gGl}$ and $1 \mathrm{gG} 3$. Ottensen et al., (1981) demonstrated that during the course of natural infection, patients with lymphatic filariasis developed high levels of $1 \mathrm{gG}$ "blocking antibodies" that could suppress or modulate $1 \mathrm{gE}$-mediated allergic reactivity to parasite antigens. These types of specific $1 \mathrm{gG}$ and $1 \mathrm{GE}$ antibodies, were reported in onchocerciasis by Ambroise Thomas (1980). Ivalions et al., (1984) detected that mice, which were injected with living microfilariae during primary or secondary infections, exhibited a high level of resistance to challenge doses. Townson et al., (1985) detected that the effects of sensitization with a range of heterologous species in mice, which had received a primary infection with $O$. cervicalis microfilariae, were significantly resistant to challenge with O.lienalis microfilariae. Immunization with uterine contents, (eggs and microfilariae) of $O$. lienalis, O. gutturosa or O.volvulus, conferred equivalent levels of protection against challenge with $O$. lienalis microfilariae. Similar result was obtained with in mice, immunized with either fresh or freeze killed (freeze inactivated) eggs of gutturosa. In conclusion, the workers found that partial resistance in mice, to Onchocerca microfilariae may be stimulated by factors yet to be determined, which are neither stage nor specie specific. Resistance to Onchocerca lienalis microfilariae in mice was conferred by egg antigens of homologous and heterologous Onchocerca specie, (Carlow and Bianco 1987), when embryonic stages of various Onchocerca species were used to stimulate resistance in CBA mice, to challenge infections with the microfilariae of Onchocerca lienalis. Comparable levels of resistance to challenge (29-37\%), were confirmed by living, freeze-killed, or sonicated organisms, administered with Freund's Complete Adjuvant. The investigators then concluded that the embryonic stages of both human and animal parasites provided a source of cross-protective antigens of value, in studies on resistance to Onchocerca microfilariae in experimental host.

Hyperglobulinaemia with elevated levels of specific antibody had been recognised in filariasis, with only the microfilariaemia patients having relatively deficient antibody responses.

Immunological detection of prepatent Onchocerciasis involves either the demonstration of released parasite products in body fluids of the infected host or of specific molecules resulting from immune responses against filarial larvae or immature adult worms. Monoclonal antibodies raised against Onchocerca antigens, have been used to demonstrate circulating antigens in sera of exposed or infected individuals. Antigen detection assays were evaluated and found to be far from optimal. False positive results due to rheumatoid factors and also false negative results, due to antibody excess, were observed. Lujan, et al (1984) in an attempt to determine the susceptibility of a heterologous filarial antigen for measuring O.volvulus antibodies compared the heterologous adult Brugia malayi and homologous Onchocerca volvulus antigens in the enzyme linked immunosorbent assay (ELISA), for onchocerciasis in Guatemala. There was a good correlation $(r=0.74)$ between the two test antigens.

Based on the above literature, the present study was aimed at using laboratory rodents as models, for immunological studies, in order to determine species specific resistance, stage specific resistance and survival rates of microfilariae and adult worms of Onchocerca volvulus and O. armillata, after initial implants or immunizations.

\section{MATERIALSAND METHODS}

\section{Sources of $O$. armillata and $O$. volvulus}

Adult worms of $O$. armillata were obtained from aortic nodules of cattle, within 20 minutes of slaughter, at the Ibadan Municipal abattoir.

Adult worms of $O$. volvulus were also obtained from subcutaneous nodules of volunteer onchocerciasis patients at Jago village in Oyo State. The site of biopsy was cleaned with methylated spirit and a small incision made. The nodule was cleared of surrounding tissue, ligated and then cut. The sight of incision was then sutured routinely.

\section{Worm Recovery from Nodules}

Initially, worm recovery was done using the technique of Albeiz Buttner and Schulz-Key (1984) by digestion with collagenase. Eventually, Akinboye's technique, which was a combination of collagenase digestion and gentle dissection with teasing, was used for worm recovery, following aseptic procedures.

Both male and female worms were recovered. The recovered worms were stored in sterile 199 medium Dubaco, until transplanted. Viability of worms was determined after microscopic examinations for motility. Only intact worms were used. 


\section{Implantation of Adult O. armillata and O.volvulus}

The experimental animals used were in bred male Swiss albino mice, with age ranges of 12 to 15 weeks and weighing about 25 to 28 gms., at the commencement of each experiment, were used for the study. Worms were surgically implanted into experimental animals within $1 / 2-1$ hour after recovery, using aseptic methods.

Each of the experimental animals was anaesthetized, using chloroform in a fume cupboard. The lower abdomen of each animal was cleaned with methylated spirit $(70 \%$ methanol), and the skin was shaved. A longitudinal incision of about $10 \mathrm{~mm}$ was made on the abdomen, into the peritoneal cavity. The worms were implanted at the ratio of one male to one female, into the peritoneal cavity of each experimental animal. The abdominal muscle of each of the animals was then sutured using catgut, while the skin was closed routinely with silk sutures. The silk suture was removed at 7 days.

Recovery of $O$. armillata and $O$. volvulus from Experimental Animals

Each group of experimental animals was examined post mortem at 7 days, 30 days or 60 days, depending on the experimental period. The nodules formed in the experimental animals were gently teased and any worm found was removed and examined thoroughly to determine whether it was still alive or dead. Statistical analysis were carried out on the results obtained to determine if there were significant differences in the survival rates.

\section{Examination of Experimental Animals for Microfilariae}

The peritoneal fluid from each of the experimental animal was obtained by rinsing out the peritoneal cavity of each animal with $1 \mathrm{ml}$ solution of acid citrate dextrose, which was then collected with a Pasteur pipette. The fluids were spun down and the precipitates obtained were examined microscopically. Smears were made for microscopic examinations.

Although the microfilariae of Onchocerca species are not blood borne, blood samples of the experimental animals were collected and examined for microfilariae as suggested by Buck et al (1969), who recommended that all blood samples should be examined for the presence of microfilariae in onchocerciasis.

Blood smears were made and examined microscopically while some blood was also haemolysed with distilled water and spun in a centrifuge. The deposits were examined microscopically for the presence of microfilariae.

Skin snips were collected from experimental animals examined for microfilariae. The skin snip was placed in $100 \mu \mathrm{l}$ of normal saline in a flat-bottomed microtitre plate, covered and incubated at $37^{\circ} \mathrm{c}$, in a water bath. These were examined at 4 hourly intervals for 24 hours.

\section{Experiment 1: An Investigation on the Effect of Primary Implantation on the Survival of Secondary Implants in Rats}

Eight adult worms of $O$. armillata were surgically transplanted into the peritoneal cavities of each of 16 rats. These were left for 60 days. A secondary implant, with another batch of 8 worms, was given to each of 12 of the experimental rats. These rats were examined at 7 and 30 days respectively,(after the second implants), for the presence of nodules and surviving worms. This experiment was repeated, using O.Volvulus. Four adult worms of $O$. volvulus were given to each of the 16 rats, for the primary implantation and subsequently, 4 worms were secondarily implanted into each of 12 of the rats, for the challenge implants. These were also examined at 7 and 30 days post implantation.

For the first control group, 12 rats were given only a primary implant of 8 worms each, of $O$. armillata and for the second control group, 12 rats were each given 4 adult worms of $O$. volvulus. The two groups of rats were examined at 7 and 30 days post implantation.

\section{Experiment II: A study of Passive Transfer of Resistance, Using Peritoneal Exudates, in Rats}

Each of 8 rats was given $1 \mathrm{ml}$ of $8 \mu \mathrm{g}$ of peritoneal exudate protein, obtained from rats, after 60-day-old transplants, of adult $O$. armillata (from Experimental 1 above), intraperitoneally. The concentration of the exudate was determined by the Biuret method and it was administered at 2 -day intervals for 10 days, in five divided doses. On the last day of injection of the exudates, 8 worms were transplanted into each of the passively immunised rats. The experiment was repeated, using $O$. volvulus with another batch of 8 rats. The peritoneal exudate used was obtained from rats after 60-day old implants of adult $O$. volvulus, (from Experiment 1 above). 4 worms were implanted into each of the 8 rats that were passively immunised with the peritoneal exudate. 
The experimental rats were later examined for the presence of nodules and adult worms, 30 days after the implantation of the worms.

In the control group, each of 8 rats was given $1 \mathrm{ml}$ of sterile $0.9 \%$ normal saline intraperitoneally, on alternate days, for 10 days. 8 Worms of $O$. armillata were later implanted intraperitoneally into each of four rats. 4 worms of $O$. volvulus were also implanted into the peritoneal cavities of the other group of 4 rats. The two groups of 4 rats were examined for nodular formation and survival of transplanted worms at 30 days.

\section{Experimental III: An Investigation of Stage Specific Resistance in Experimental $O$. armillata and $\boldsymbol{O}$. Volvulus Infection in Rats}

Two groups of 8 rats were immunised with either adult worm homogenate of $O$. armillata or $O$. volvulus homogenate, emulsified in complete Freund's adjuvant, at 7 days intervals on 4 occasions. $8 \mu \mathrm{g}$ protein of $O$. armillata or $O$. volvulus homogenate was administered each time. The immunised rats were further divided into 2 groups (A and B) as further described below:

Group A: 4 rats, immunised with $O$. armillata homogenate, were given 5,000 microfilariae of $O$. armillata by intraperitoneal injection, 30 days after the starting dose of immunization with adult worm protein. Also 4 rats, immunised with $O$. volvulus homogenate, were given 5,000 microfilariae of $O$. volvulus by intraperitoneal injection, 30 days after starting immunization with adult worm homogenate. The survival of microfilariae was monitored for 130 days.

Group B: Four rats which were immunised with $O$. armillata homogenate were each implanted with 8 adult worms of $O$. armillata, $a 30$ days after the first dose of immunisation. Similarly, 4 rats, immunised with $O$. volvulus homogenate, were each implanted with 4 adult worms of $O$. volvulus (30 days after the first dose of immunisation). The survival rates of the implanted worms were observed.

Group C: This group served as the control for the experiment. 5,000 live microfilariae of $O$. armillata were injected intraperitoneally into 8 naive rats. The survival of the microfilariae was monitored in 4 rats for 130 days and 8 adult worms were transplanted into each of the other 4 rats at 30 days. 5,000 live microfilariae of $O$. volvulus were also injected intraperitoneally into another 8 naive rats. The survival of the microfilariae was monitored in 4 rats for 130 days while 4 adult worms were transplanted into each of 4 of the other rats at 30 days. The survival rates of the implanted adult worms were monitored. $1 \mathrm{ml}$ of $0.9 \%$ saline was also injected into the peritoneal cavities of each of 8 rats. 8 adult worms of $O$. armillata were implanted into the peritoneal cavities of 4 of these rats while 4 adult worms of $O$. volvulus were also implanted into the peritoneal cavities of each of the other 4 rats, at 30 days after the injection of saline. The rats were necropsied at 30 days post implantation of $O$. armillata and $O$. volvulus. The survival rates of these implanted worms served as the control for the implanted $O$. armillata and $O$. volvulus above.

\section{Experiment IV: The Effect of Corticosteroid on Survival of Adult $O$. armillata and $O$. volvulus}

Each of 18 rats was given injections of $1.8 \mathrm{mg}$ of dexamethasone intraperitoneally, on alternative days for 10 days. 8 adult worms of $O$. armillata were implanted into each of 9 of the rats. The administration of dexamethasone was continued daily for another 7 days after the implantation of adult $O$. armillata. The survival rate of the implanted worms was determined at 30 days. Similarly, 9 rats given dexamethasone $(1.8 \mathrm{mg} / 90 \mathrm{gm}$ body weight) were each implanted with 4 adult worms of $O$. volvulus. The administration of dexamethasone was also continued for another 7days, post implantation. The survival rate of the implanted worms was determined at 30 days post implantation. The control for this experiment was set up as in Experiment II above, where the injection of $0.9 \%$ normal saline injection was substituted for dexamethasone.

\section{Experiment V: An Investigation on Specie-Specific Resistance on Onchocerciasis}

The experimental rats were divided into the following groups:

Group 1: 8 rats were immunised with $O$. armillata adult worm homogenate (as previously described in Experiment III). The rats were implanted with 4 adult worms of $O$. volvulus each, at 30 days post immunization and were all necropsied and examined for surviving worms after another 30 days after the secondary implantation with $O$. volvulus

Group II: 8 rats were immunised with adult worms homogenate of $O$. volvulus every 7 days for 28 days (as described in Experiment III). The rats were 
implanted with adult worms of $O$. armillata $(8$ worms each, as described in Experiment III). The rats were examined for surviving worms, 30 days after the secondary implantation.

\section{RESULTS}

Experiment 1: The Influence of Primary Infections on the Establishment of Secondary Implants with $O$. armillata and $O$. volvulus In Rats, 7 Days PostImplantation

In animals necropsied and examined 7 days after the secondary implant with $O$. armillata, nodules were formed but no living worm was recovered from the nodules (table 1). Remains of degenerating adult worms only, were found in the nodule. In the control group, one nodule was formed in each animal and $78 \%$ (or 25 out of 32 ) of all the implanted worms of $O$. armillata had a significant influence on the survival rate of a second batch of implanted worms of the same species, $\left(X^{2}=64, \mathrm{df}=1, \mathrm{P}<0.001\right)$.

In the experimental rats which were implanted with $O$. volvulus, $13 \%$ (2 out of 16 ) of the second implanted worms survived for up to 7 days (Table 1). The surviving worms were recovered from nodules measuring between 0.5 to $0.8 \mathrm{~mm}$ in diameter. However, in the control group, $69 \%$ of the worms implanted were still alive on the 7 th day.

Based on these results, primary implanted worms significantly reduced the survival rate of the secondary implanted worms $\left(\mathrm{x}^{2}=32, \mathrm{df}=1, \mathrm{P}<0.001\right)$.

\section{Days Post-Implantation}

With $O$. armillata, no worms were recovered alive in nodules obtained in rats necropsied 30 days after a secondary implant with homologous series (Table 1). In the control group, nodules measuring between 1 to $2 \mathrm{~mm}$ were recovered. $59 \%$ (or 19 out of 32 ) living worms were recovered (Table 1.)

In the rats implanted with $O$. volvulus, nodules measuring approximately 0.2 to $0.6 \mathrm{~mm}$ in diameter were formed. None of the worms were recovered alive. However, in the control group, $56 \%$ (or 9 out of 16 ) of the implanted $O$. volvulus survived. (Table 1).

There was a significant difference between the survival rate of worms and the primary implants of adult worms. $\left(\mathrm{X}^{2}\right.$ $=5.2, \mathrm{df}=1, \mathrm{P}<0.2)$.

TABLE 1: The Influence of a Primary Implant of $O$. armillata and $O$. volvulus on The Establishment of a Secondary Implant with Homologous Species

\begin{tabular}{|c|c|c|c|c|}
\hline $\begin{array}{l}\text { Species } \\
\text { of Adult } \\
\text { worm }\end{array}$ & \multicolumn{3}{|c|}{7 days } & \multirow[b]{2}{*}{ No. of Wor } \\
\hline $\begin{array}{l}\text { O. } \\
\text { Armillata }\end{array}$ & $\begin{array}{l}\text { No. of Worms } \\
\text { Implanted }\end{array}$ & $\begin{array}{l}\text { No. of Worm } \\
\text { Surviving }\end{array}$ & $\begin{array}{l}\% \text { of Worms } \\
\text { surviving }\end{array}$ & \\
\hline Test & $\begin{array}{l}32 \\
(8,8,8,8)\end{array}$ & $\begin{array}{l}0 \\
(0,0,0,0)\end{array}$ & 0 & $\begin{array}{l}32 \\
(8,8,8,8)\end{array}$ \\
\hline Control & $\begin{array}{l}32 \\
(8,8,8,8)\end{array}$ & $\begin{array}{l}25 \\
(6,5,7,7)\end{array}$ & 78 & $\begin{array}{l}32 \\
(8,8,8,8)\end{array}$ \\
\hline $\begin{array}{l}\begin{array}{l}O . \\
\text { volvulus }\end{array} \\
\text { Test }\end{array}$ & $\begin{array}{l}16 \\
(4,4,4,4)\end{array}$ & $\begin{array}{l}2 \\
(0,0,1,1)\end{array}$ & 13 & $\begin{array}{l}16 \\
(4,4,4,4)\end{array}$ \\
\hline Control & $\begin{array}{l}16 \\
(4,4,4,4)\end{array}$ & $\begin{array}{l}11 \\
(2,5,2,2)\end{array}$ & 69 & $\begin{array}{l}16 \\
(4,4,4,4)\end{array}$ \\
\hline
\end{tabular}

(No of worms implanted or recovered in each individual rat was as shown in brackets, each figure represents worms in one rat) 


\section{Experiment II: Effect of Passively Transferred Peritoneal Exudate on Survival of $O$. armillata and $O$. volvulus Infections}

In rats immunised with peritoneal exudate, and subsequently implanted with $O$. armillata worms, nodules measuring about $2-2.5 \mathrm{~mm}$ in diameter were formed 30 days after implantation of worms. $59 \%$ of the implanted worms were recovered alive, (Table 2), while the others were either degenerated or absorbed.

Nodules were also formed in the control rats where $69 \%$ (or 22 out of 32) of the implanted worms were recovered alive. When the result of the test and the control were compared, it was concluded that the peritoneal exudate had no significant effect on the survival of implanted worms. $\left(\mathrm{X}^{2}=1.1, \mathrm{df}=1, \mathrm{P}>0.05\right)$.

On the other hand, using O. volvulus, no life worms were recovered in nodules 30 days post implantation in rats immunised with peritoneal exudate. Pieces of degenerated worms were present in some of the nodules. However, in the control group, $44 \%$ ( 7 out of 16 ) worms survived. (Table 2). Immunisation with peritoneal exudate had a significant effect on the survival of $O$. volvulus in rats $\left(\mathrm{X}^{2}=27, \mathrm{df}=1, \mathrm{P}<0.001\right)$.

TABLE 2: The Effect of Passively Transferred Peritoneal Exudate On Survival of $O$. armillata and $O$. volvulus at 30 Days

(number of worms implanted or recovered in each individual rat was as shown in brackets, each figure represents one rat)

\section{Experiment III: Stage Specific Resistance In O. armillata and $O$. volvulus \\ Group A}

In the group of rats immunised with adult $O$. armillata homogenate and injected with microfilariae of $O$. armillata, microfilariae were not detected in either the blood or the skin snips of the rats, but live microfilariae were detected in the peritoneal exudate of the rats for up to 20 days after the injection of microfilariae. The microfilariae were not active and so they were believed to be dead. Microfilariae were not detected in body fluids or skin snips 60 days after the injection with microfilariae of $O$. armillata.

In the group of rats immunised with adult worm homogenate of $O$. volvulus and injected with microfilariae of $O$. volvulus, live microfilariae were detected in the skin snip of only one of the rats. There were no microfilariae present in the blood sample of any of the rats. Live microfilariae were detected in the peritoneal exudate for 30 days. None active microfilaria, which were believed to be dead were still present in the peritoneal exudate for up to 60 days.

\section{Group B}

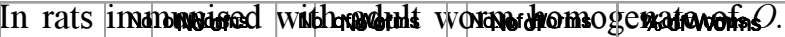

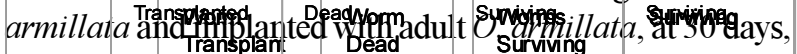
the survival eate of the worms was $38 \%$ (12 out of 32$)$,

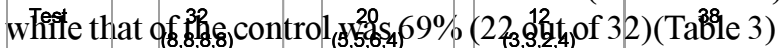

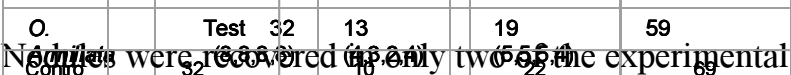
arimals that 8 8.

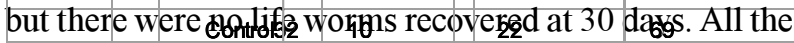
implanted wortis,8, survival rate of $44 \%$ (Table 4 ). The comparison of the test and control, ung ${ }_{16}$, indicated that there was a

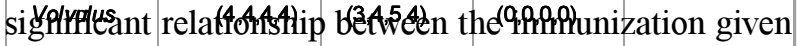
to the animals and the survival rate of the implanted worms. \begin{tabular}{|c|l|l|l}
$\left(\mathrm{x}^{2}=2, \mathrm{df}=\right.$ & $\begin{array}{r}\left.1, \mathrm{pe}_{(4,4,4,4)}\right) \\
(2,3,2,2)\end{array}$ & $\mathbf{( 1 , 2 , 4 , 0 )}$ & 44
\end{tabular}

TABLE 3: The Effect of Immunization with Adult Worm Homogenate of $O$. armillata On Transplanted Adult O. armillata at 30 Days

(Number of worms implanted or recovered in each individual rat was as shown in brackets, each figure represents one rat) 
TABLE 4: The effect of immunization with adult worm homogeneate of $O$. volvulus on transplanted worms of O. volvulus at 30 days

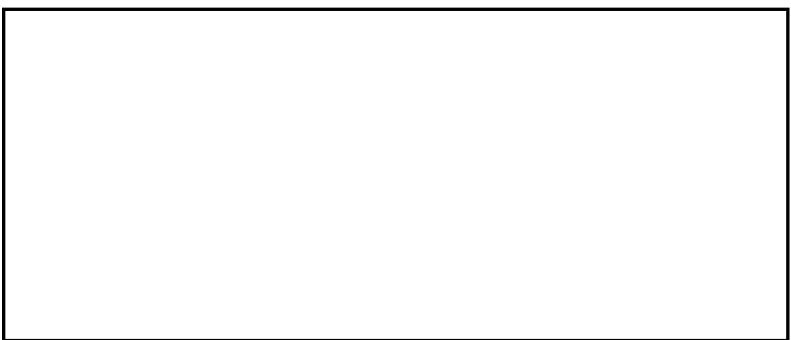

(Number of worms implanted or recovered in each individual rat was as shown in brackets, each figure represents one rat)

\section{Group C}

This group served as the control group. In the rats that were injected with microfilariae of $O$. armillata only, life microfilariae of $O$ armillata were not detected in the blood. Microfilariae were detected in the skin snips of one rat at 10 days and 20 days. Life microfilariae were detected in the peritoneal exudate of the animals for up to 40 days. No active microfilariae were detected in the peritoneal exudate for up to 60 days.

In rats injected with microfilariae of $O$. volvulus only, life microfilariae were detected in the skin of 3 animals at 20 days and were also detected in the peritoneal cavities for up to 50 days. None active microfilariae were detected at 80 days, these are believed to be dead.

Rats that were injected with microfilariae of $O$. armillata and implanted with adult worms of $O$. armillata were examined at 30 days. The percentage survival of adult worms was $53 \%$ (17 out of 32), while that of the control was $69 \%$ (22 out of 32 ) (Table 5). The statistical analysis showed that there was no significant relationship between the immunization with microfilariae and the survival rate of O. armillata.

TABLE 5: The effect of immunization with microfilariae of $O$ armillata on transplanted adult worms of $O$. armillata at 30 days

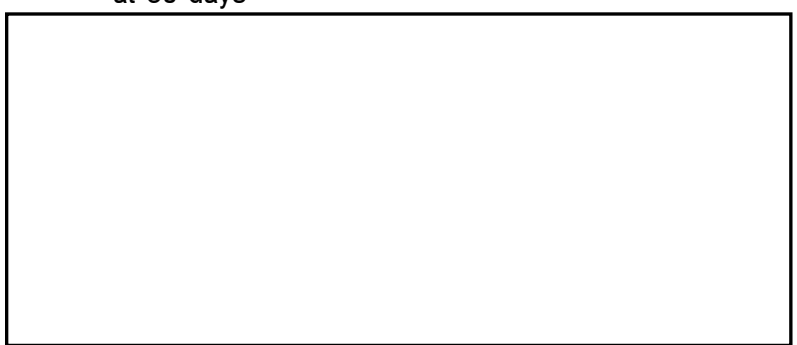

(Number of worms implanted or recovered in each individual rat was as shown in brackets, each figure represents one rat)
In the group of rats injected with $0.9 \%$ saline and implanted with $\underline{O}$. armillata, the survival rate was $69 \%$ (22 out of 32 ) at 30 days. In the group of rats injected with physiological saline and implanted with $O$. volvulus, the survival rate was $44 \%$ ( 7 our of 16$)$ at 30 days.

Nodules were formed in the rats that were surgically transplanted with adult worms after an initial injection with microfilariae. When these were examined at 30 days, there was $38 \%$ survival of the $O$. volvulus worms transplanted. (Table6). The control had a $44 \%$ survival rate. When the survival rates of the test and the control were compared, it was detected that the injection of microfilariae had no significant effect on the transplanted worms. $\left(\mathrm{x}^{2}=0.4, \mathrm{df}=\right.$ $1, \mathrm{P}>0.005)$.

TABLE 6: The effect of immunization with microfilariae of $O$. volvulus on transplanted adult worms of $O$. volvulus on transplanted adult worms of $O$. volvulus at 30 days.

Test(Number Test
rat was as shown in brackets, each figure represents one rat)

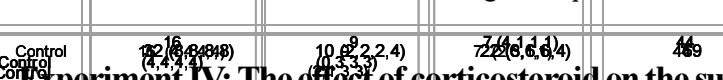

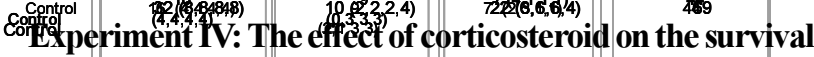
rate of implanted $O$. armillata and $O$. volvulus

Only 5 out of the 9 rats used for this experiment survived after the administration of steroid. 3 out of the five surviving rats were examined at 30 days and the other two at 60 days post-implantation of $O$. armillata. The average survival rate for the 3 rats at 30 days was $79 \%$ (19 out of $24)$, while the average survival rate at 60 days was $13 \%$ (2 out of 16). (Table 7). In the control group where only physiological saline was injected, the survival rate was $69 \%$ (22 out of 32 ), at 30 days post implantation and $3 \%$ ( 1 out of 32) at 60 days post implantation.

The administration of corticosteroid did not have a significant effect on the survival of $O$. armillata adult worms at 30 days. At 60 days, corticosteroid appeared to promote the survival of worms, unlike in the control rats $\left(\mathrm{x}^{2}=3, \mathrm{df}=1, \mathrm{P}>0.5\right.$. 
With $O$. volvulus, out of the surviving rats that were given corticosteroid, an average of $69 \%$ (11 out of 16 ) of the worms implanted in 4 rats survived at 30 days while $44 \%$ (7 out of 16) survived in the control. Also, 25\% (2 out of 8 ) survived at 60 days while $6 \%$ ( 1 out of 16 ) survived in the control (Table 7). there was no significant difference between the implants of animals immunised with adult worm homogenate of $O$. armillata and the survival rate of the subsequently implanted $O$. volvulus worms $\left(\mathrm{x}^{2}=0.4, \mathrm{df}=1, \mathrm{P}>0.05\right)$.

TABLE 7: The effect of corticosteroid on the survival of adult O. armillata and O. volvulus

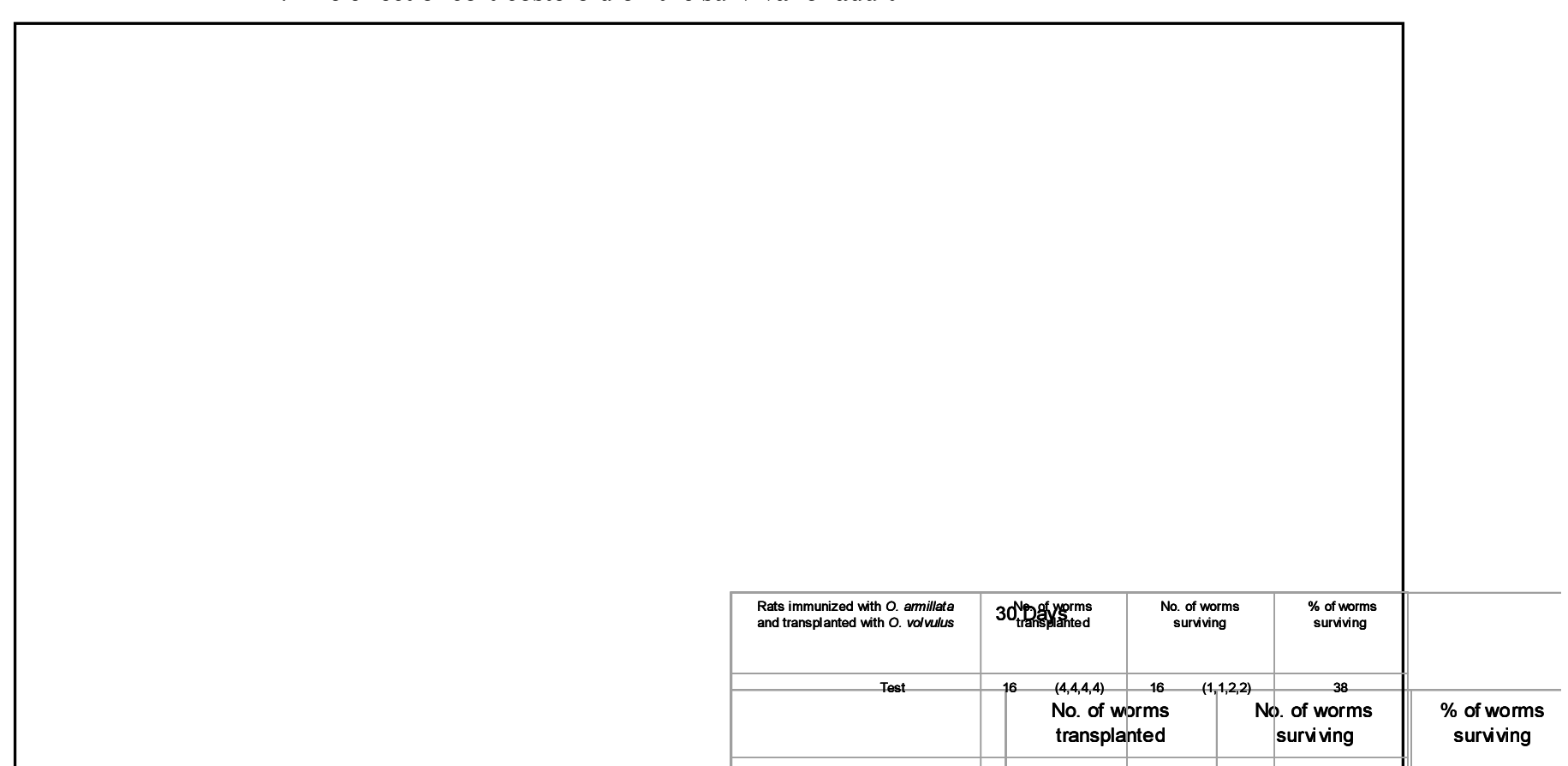

(Number of worms implanted or recovered in each individual rat was as shown in brackets, each figure feperents one rat)

Corticosteroid appeared to increase the survival rate of the implanted $O$. volvulus worms significantly at 30 days $\left(\mathrm{x}^{2}=6.3, \mathrm{df}=1, \mathrm{P}<.01\right)$. Corticosteroid also appeared to increase the survival rate of the implanted worms at 60 days although the surviving worms were too few for detailed analysis.

\section{Experiment V: Investigation of Specie Specific Resist- ance in Onchocerciasis}

\section{Group I}

In this experiment where rats were immunised with $O$. armillata adult worm homogenate, and later transplanted adult worms of $O$. volvulus $38 \%$ (6 out of 16) of the implanted worm survived and formed nodules at 30 days. The control group had a survival rate of $44 \%$ (7 out of 16) (Table 8). When the result of the test was compared with the control,

\begin{tabular}{l|r|r|r}
\hline O. armillata & Test & 24 & 19 \\
\hline
\end{tabular}

Rats immunized with O.volvulus

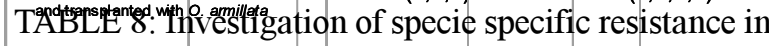

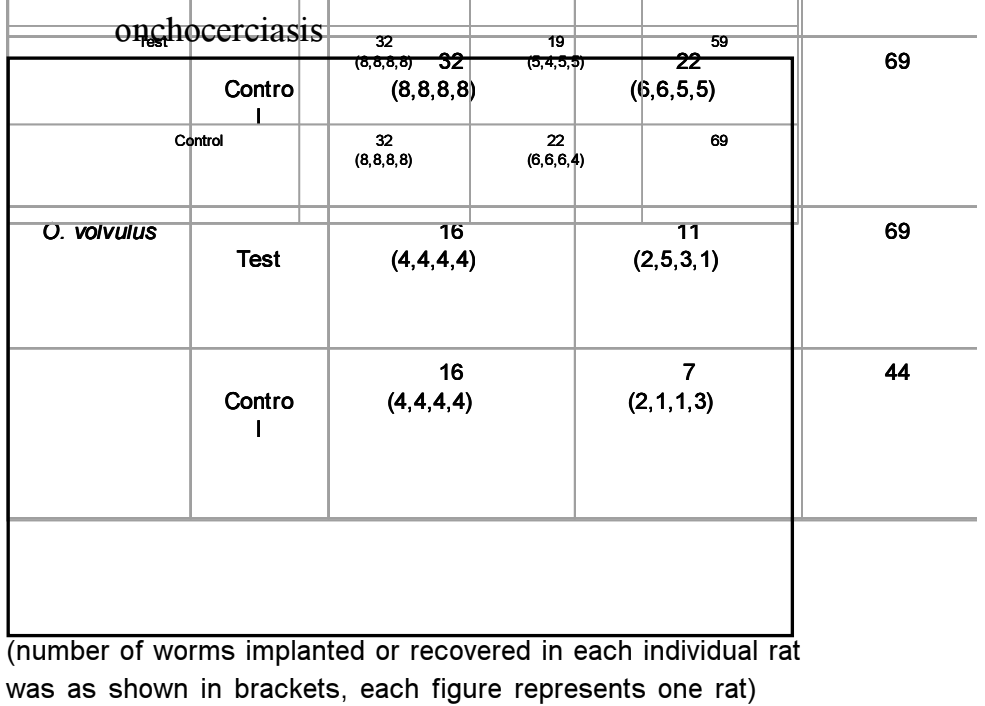




\section{Group 11B}

Nodules were formed in the experimental rats immunized with $O$. volvulus adult worm homogenate, and implanted with $O$. volvulus, it did not have any significant effect on the survival rate of the implanted worms. $\left(\mathrm{x}^{2}=1.0, \mathrm{df}=1\right.$, $\mathrm{P}>0.05)$. The above results show that immunization with one species of Onchocerca does not influence the survival rate of another species.

\section{DISCUSSIONAND CONCLUSION}

The finding of this investigation are very important because, the survival of adult Onchocerca in infected host is crucial in determining the clinical outcome of diseases in several hosts. In onchocerciasis, high levels of antibody and cell-mediated immunity have been demonstrated (Ottensen 1984; Gallin et al., 1988). For instance, opsonic antibody promote adherence of eosinophils and neutrophils to $O$. volvulus and lead to killing of such microfilariae (Greene 1987). Duke and More (1968) noted plateaning of microfilarial density in the skin after 20-40 years, this is an observation which relfects mirofilarial immunity.In this study, it has been shown that immunization or implantation of rats with $O$. volvulus and $O$. armillata hindered the survival of secondary implantation, while cortisone therapy promoted the survival of worms. Cortisone mediated immune suppression may be due to either a direct action on splenic lymphocytes or an indirect action on the ability of macrophages to respond to lymphomas (Collins and Dees, 1986). Cortisone treatment may also abrogate monocyte effector mechanism by reducing phagocytosis and respiratory burst activity (Masur et al., 1982).

Cortisol and its derivatives are believed to suppress delayed type hypersensitivity (Weston et al., 1973) and cortisol is known to decrease serum IgG concentration in man (Butler and Rossen 1973).

Thus immunity to Onchocerca species has been amply demonstrated in this study. However, Duke (1968) showed that individuals in the field can be reinfected after Suramin treatment, suggesting that immunity is neither effective nor complete in onchocerciasis. In other filariasis, partial immunity has been shown to occur in $A$. vitae, in jirds and D. immitis infection in dogs (WHO, 1987). Introduction of host-immunity in A. vitae appear to be host-specific since jirds, but not hamsters, had demonstrable protective immunity to $A$. vitae. The protective antigens seem to be parasite E-S products released by life worms.
Some hypotheses have been suggested to explainthe paradox of immunity in the presence of life worms. These are that suppression of immunity in onchocerciasis and other filariasis occur either in a specific manner (Ottensen et al, 1977; Piessens et, al, 1980) or in a non-specific manner through block anti-bodies, (Hussain et al, 1981). This study shows that an animal model can be used to further investigated mechanisms of resistance in Onchocerciasis.

WHO (1976) stated that there was little evidence from animal studies that the resistance conferred by vaccination is directed against adult worms. However, the results obtained in this work show a reduction in the survival rates of worms, in rats with primary implants, or vaccinated with worm extracts.

This model is valuable for short time maintenance of both adult $O$. armillata and $O$. volvulus. It is also useful for immunological and chemotherapeutic studies of the two species of Onchocerca worms.

Based on the results obtained, the following conclusions can be made:

1. The administration of steroid increases the survival rate of implanted worms of $O$. volvulus and $O$. armillata in the rat.

2. There is stage specific resistance in $O$. volvulus and $O$. armillata.

3. There is specie specific resistance in $O$. volvulus and $O$ armillata.

\section{REFERENCES}

Albeiz, E.J., Bultner, D.W., Schulz-Key, H. (1984): Studies on nodules and adult Onchocerca volvulus during a nodulectomy trial in hyper endemic villages in Liberia and Upper Volta II: Comparison of the microfilaria population in adult nodule carriers. Tropical Medicine and Parasitoloty. 35 (3) 163-166.

Ambroise-Thomas, P. (1980): Filariasis In: Immunological Investigation of Tropical Parasite Diseases. V. Houba (Editor) New York: Churchill Livingston, pp. 84 - 103.

Buck, A.A. (1974): Systemic onchocerciasis. In onchocerciasis symptomatology, pathology, diagnosis, A.A. Buck (ed.). World Health Organisation, Geneva, pp. $\quad 14-15$. 
Buck, A.A., Anderson, R.I., Kwata, K. and Huckocock, J.C. (Jnr.), (1969): Onchocericasis: Some new epidemiological and clinical findings. American Journal of Tropical Medicine and Hygiene, 18, 217 230.

Butler, W.T., Rossen, R.D. (1973): Effects of corticosteroids on immunity in man. Decreased serum IgG. Concentration caused by 3 or 5 days of high doses of Methylprednisolone. Journal of Clinical Investigations, 52: 2629.

Carlow, C.K.S. and Binaco, A.E. (1987): Resistance to Onchocerca lienalis microfilariae in mice conferred by egg antigens on homologous and heterologous Onchocerca species. Parasitology, 94, 485 - 496.

Collins, W.E., and Dees (1986): The use of fixed tissue sections to determine antibodies to Onchocerca volvulus in a fluorescent antibody test. Journal of Parasitology, 65, 827 - 829.

Connor, D. and Neafie, C., (1976): Pathology of Tropical and Extraordinary Diseases, 11, pg. 360. Pub. Armed Forces Institute of pathology, Washington, D.C., U.S.A.

Cupp,E.W.,(1996): Black flies and the agents they transmi in"the Bilogy of Disease vectors",Ed.Beaty,B.J.and Marquardt.,W.C.,University Press of Colorado.

Duke, B.O.L. (1968): Reinfections with Onchocerca volvulus in cured patients exposed to continuing transmission. Bulletin of World Health Organisation. 39, $307-309$.

Duke, B.O.L., Moore, P.J. (1968): The contribution of different age groups to the transmission of Onchocerciasis in Cameroon forest village.Transactions of the Royal Society of Tropical Medicine Hygiene, 62, 22 - 28.

Gallin, M., Edmonds, K., Jerrold, J.E., Erttmann, K.D., White, A.T., Newland, H.S., Taylor, H.R. and Greene, B.M. (1988). Cell-mediated immune response in human infection with Onchocerca volvulus. The Journal of Immunology. 140, 1999 - 2007.

Gibson, D.W., Connor, D.H., Brown, H.L., Funglsang, H., Anderson, J., Duke, B.O.L., Buck, A.A. (1976). Onchocercal dermatitis ultra structural studies of microfilariae and host tissue, before and after treatment with diethylcarbamizine Hetrazan. American Journal of Tropical Medicine and Hygiene, 22, 74 - 87.
Godon, R.M., and Laviopierre, M.M. (1978): Entomology for Students of Medicine. Blackwell Scientific Publications, Oxford, London, Edinburgh, Melbourne. 5th Ed. Pg. 144.

Greene, B.M. (1987): In vitro antifilarial killing mechanisms. W.H.O. Report of the thirteenth meeting of the scientific working group on filariasis. 6 - 9 April. Pg. 8.

Henson, P.M., Mackenzie, C.D. and Spector, W.G. (1979): Inflammatory reactions in onchocerciasis: a report on current knowledge and recommendations for further study. Bulletin of the World Health Organization. 57, $667-82$.

Hussain, R., Hamilton, R.G., Kumaraswami, V., Adikinson, (Jr.), N.F. and Ottensen, E.A. (1981). IgE responses in human filariasis 1. Quantitation of filarial-specific $1 \mathrm{gE}$. Journal of Immunology, 127, 1627 - 1629.

Ivalisons, S., Bianco, A.E., Doeuhoff, M.J., and Maller, R., (1984): Immunity to Onchocerca lienalis microfilariae in mice. Tropical Medicine and Hygiene. 185 - 202.

Lujan, R., Collins, W.E., Stanfill, P.S., Campbell, C.C., Collins, R.C., Huong, A.Y. (1984): Comparison of heterologous adult Brugia malayi and homologous Onchocerca volvulus antigen in the enzyme linked immunosorbent assay (ELISA) for Guatemalan onchocerciasis. Journal of Parasitology, 70, 385 - 390.

Mackenzie, C.D. (1980): Aspects of the generation, circulation and function of eosinophil leucocytes in helminthic infections. Volume 1. Thesis: Roy coll. of Vet. Surgeon, London, 153.

Masur, H., Murray, H.W., and Jones, T.C., (1982). Effect of hydrocortisone on macrophage response to lympokine. Infectious Immunology. 35: 709 - 714.

Muller, R. (1978): Identification on Onchocerca, (pp. 175 - 196). Department of Tropical Helm London School of Hygiene and Tropical Medicine, London WC1E 7HT, $175-206$.

Noble, E.R. and Noble, G.A. (1976): The Biology of Animal Parasites. Pg. 321. Henry Kinspton Publishers, London.

Ogundipe, G.A., Ogunrinade, A.F., Akpavie, S.O. (1984): Prevalence, gross lesions and histopathology of aortic onchocerciasis in Nigeria cattle. The Veterinary Quarterly, 6, No. 2, 85 - 89. 
Ogunrinade, A.F., Kale, O.O. and Piessens, W.F. (1985): Comparison of Polypeptide and glycoprotein antigens of Onchocerca volvulus with those of the related bovine (Onchocerca armillata). (Unpublished observation).

Ottensen, E.A. (1984): Immunological aspects of lymphatic filariasis and onchocerciasis in man. Transactions of the Royal Society of Tropical Medicine and Hygiene, 78, (Supplement 9 - 18.

Ottesen, E.A., Kuwaraswami, V., Paranjape, R., Poindexter, R.W. W. and Tripathy, S.P. (1981): naturally occurring blocking antibodies modulate immediate hypersensitivity responses in human filariasis. Journal of Immunology, 127, 2014 - 2020.

Ottensen, E.A., Weller, P.F., Heck, L. (1977): Specific Cellular immune irresponsiveness in human filariasis. Immunology, 33, 413 - 421.

Piessens, W.F., Machenzie, C.D. (1982): Immunology of lymphatic filariasis and onchocerciasis. In Immunology of Parasitic Infections (eds. S. Cohen, K.S. Warren). 2nd Ed. Oxford, Blackwell, 622 - 53.

Piessens, W.F., Partono, F. (1980): Host Vector parasite relationships in human filariasis. In seminars In: Infectious Diseases (Eds. L. Weinstein and B.N. Fields). Stustgard: G. Thieme, 13, 131-152.

Portono, J.K., Brilton, S. and Ash, L.R. (1976): Brugia Pahangi depressed mitogen reactivity in filarial infections in the jirds. Meriones unguiculatus. Experimental Parasitology, 40, 438 - 466.

Sisley, B.M., Machenzie, C.D., Steward, M.W., William, J.F., O’Day, J., Luty, A.J., Braga M.el Sheikh (1987): H. Associations between clinical disease circulating antibodies and C'o binding immune complexes in human onchocerciasis. Parasite Immunology, 9 (4) $447-463$

Stevenson, D. (1987) : Davey and light body's 'the control of disease in the tropics. Aldeen Press, London H. K. Lewis \& Co.Ltd. Pg 209-210.

Townson, S., Nelson, G.S., and Bianco, A.E. (1985): Immunity to Onchocerca linalis microfilaria in mice II. Effects of sensitization with a range of heterologous species. Journal of Helminthology, 59, 337 - 46
Weiss, N. (1978): Dipetalonema vitae. In vitro blastogenesis of hamster spleen and lymph node cells to phyto haemaglutinin and filarial antigens. Experimental Parasitology, 46, 283 - 299.

Weiss, N. Specicer, F. and Hussain, R. (1981): 1GE antibodies in human onchocerciasis. Application of newly developed radio allergosobent test (RAST). Acta Tropica, 38, $353-362$.

Weston, W.L., Claman, H.N., Krueger, G.G. (1973): Site of action of cortisol in cellular immunity. Journal of Immunology, 110: 880 .

W.H.O. (1973): Onchocerciasis: Control in the Volta River Basin Area. Report of the Preparatory Assistance Mission to the government of Dahomey, Ghana, Ivory Coast, Mali, Niger, Togo, Upper Volta, Geneva, Pg. 17.

W.H.O. (1976): Expert Committee on epidemiology of Onchocerca raibalis: Technical Report Series, No. 597.

W.H.O. (1978): Scientific Working Group on Onchocerciasis schistosomiasis. W.H.O. regional office for Africa, Brazzaville, pg. 7.

W.H.O. (1982): The pathogenesis and treatment of ocular onchocerciasis: Report of the Eighath Meeting of the Scientific working group on Filariasis in collaboration with the programme for the prevention of Blindness, pg. 9. Geneva, 20 -22, October.

W.H.O. (1987): Protective immunity and vaccination in Onchocerciasis and lymphatic filariais. Report on the Thirteenth Meeting of the Scientific Working Group on Filariasis. 